\title{
Numerical investigation on the effect of inlet conditions on the oblique shock system in a high-speed wind tunnel
}

\author{
Mohammad Reza Heidari", Mahdi Karami, Fata Mohammadi Fard \\ Islamic Azad University, Parand Branch, Tehran, IRAN \\ Email address: \\ heidarimr@piau.ac.ir(M. R. Heidari),mahdykaramy@yahoo.com(M. Karami), fata.aero@gmail.com(F. M. Fard)
}

\section{To cite this article:}

Mohammad Reza Heidari, Mahdi Karami, Fata Mohammadi Fard. Numerical Investigation on the Effect of Inlet Conditions on the Oblique Shock System in a High-Speed Wind Tunnel. American Journal of Physics and Applications. Vol. 1, No. 3, 2013, pp. 91-98. doi: 10.11648/j.ajpa.20130103.16

\begin{abstract}
The Present study is conducted to find out the best inlet conditions for designing process of a high-speed wind tunnel. The inlet conditions are appropriate for the cases that the real nozzle has not been calculated and downstream parts designers (such as test section and diffuser designers) need a reasonable upstream flow condition for their calculations. Inlet boundary condition of each case has been specified by User-Defined Function. The process of User-Defined Function (UDF) specification is time-consuming and is based on a try and error method. Several cases of inlet conditions have been designed and examined. The comparison of results is shown in terms of cross-sectional and axial distributions of parameters and contours of Mach number and static pressure. Finally, the $1.2 \mathrm{~m}$-length Ogive curve with 0.5 degree initial angle and after that, the case of $1.2 \mathrm{~m}$-length ducthave been chosen as the equivalent nozzles.
\end{abstract}

Keywords: High-Speed Flow, Wind Tunnel Design, Nozzle Curve Calculations, Computational Fluid Dynamics, Equivalent Nozzle

\section{Introduction}

Current and expected developments in space transportation have led to growing interest in new high speed aerospace vehicles. Several expendable and partially or fully reusable concepts are discussed or already planned. These new vehicles require essential improvements over current vehicles in order to ensure economic viability and to fulfill mission and safety constraints. Therefore, a close interaction of all involved disciplines as well as the optimal use of all technical potentialities is necessary. Simultaneously, design cycle times have to be reduced. The size and complexity of this problem has led to growing importance of numerical methods for design and optimization. Computational Fluid Dynamic (CFD) is a strategic tool which isenable to reduce dramatically the design and development time required for new vehicles. A number of developments have contributed to this situation: increased robustness of CFD codes, lower computational costs, improvements in hardware as well as grid generation and more user-friendly post-processing tools [1].

The results of CFD codes have a lot of uncertainty sources, such as numerical algorithm uncertainty, truncation error, error in flow modeling, etc. Therefore, it is necessary to validate the numerical data with a physical test (i.e., experimental results). High-speed wind tunnels are the most useful facilities that provide researchers a good vision about high-speed flow regimes. These apparatuses not only can simulate the flight conditions on the earth, but also are able to validate the data resulted from numerical approach of CFD codes.

In 1961, an experimental study on the force and heat characteristics of HB-2 model in Mach numbers from 2 to 5 was conducted by Deem that included the effect of shock impingement [2]. Another work was carried out by Gray and Lindsay in 1964 to study the force aerodynamic characteristics of HB-1 and HB-2 in both supersonic and hypersonic regimes. In this investigation, Mach number varies from 1.5 to 8 while the angle of attack differs from $-2^{\circ}$ to $15^{\circ}$ to investigate the effect of these parameters on the aerodynamic characteristics of the mentioned models [3].In 2005, two practical tests in 1.27 Mach wind tunnel and high enthalpy shock tunnel were done by JAXA researchers that included force and heat transfer measurements [4].

Although there are some methods to design nozzle curve of wind tunnel (e.g. method of characteristics [5]), unfortunately there is not any specific method that 
describes a step-by-step algorithm for designing diffuser part of a wind tunnel and the diffuser geometry should be extracted by working on an existing diffuser and modifying it by a try and error procedure to meet the goal characteristics [6].

Advanced missiles and shuttles are always faced to high-speed flow that is characterized by especial properties such as small shock stand-off distance, entropy layer, viscous interactions and high temperature conditions that make the gas ionized. Since the mission of high-speed flying vehicles are always secret and strategic, it is more important to find the fly conditions before flying. One of the most effective facilities to study the real statements of fly condition is the wind tunnel. Nozzle is a basic part of any wind tunnel that provides favorable high-speed flow (ejected into test-section) for testing of the model. Since the calculation of nozzle curve for any Mach number is very difficult and time-consuming, the designers of test-section and diffuser need an equivalent nozzle that can provide a similar flow to real nozzle for calculating the flow parameter downstream of the nozzle. The design process and assessment of equivalent nozzle samples is focused in the present manuscript.

\section{Problem Description}

The schematic view of the computational domain of considered wind tunnel is shown in Fig. 1. It consists of divergent part of nozzle, test section and diffuser. The aim of this investigation is to omit the nozzle part and replace it with the best designed equivalent part called equivalent nozzle. To reach this purpose, we need to define inlet boundary conditions with a user-defined function (UDF). The equivalent nozzle always has smaller size comparing with the main nozzle. Seven cases of different equivalent nozzles are simulated in the present study as follows:

Case-1: Real nozzle (with about $4.7 \mathrm{~m}$ length).

Case-2: A cut-off part of real nozzle at $1.2 \mathrm{~m}$ from the outlet of it, with exported data from the cut-off cross-section.

Case-3: A cut-off part of real nozzle at $1.2 \mathrm{~m}$ from the outlet of it, with UDF.

Case-4: A 1.2 m-length Ogive curve with 0.5 degree initial angle.

Case-5: A 1.2 m-length Ogive curve with 1.5 degree initial angle.

Case-6: A 0.5 m-length Ogive curve with 0.5 degree initial angle.

Case-7: A 1.2 m-length duct.

Fig. 2 depicts the different curves of mentioned cases (the horizontal and vertical measures are not in a same scale).

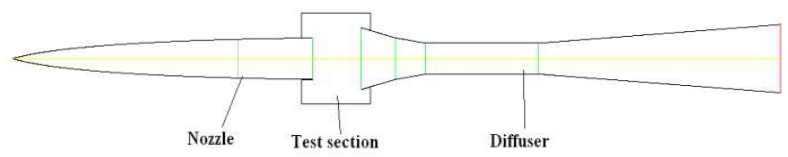

Figure 1. Schematic view of computational domain

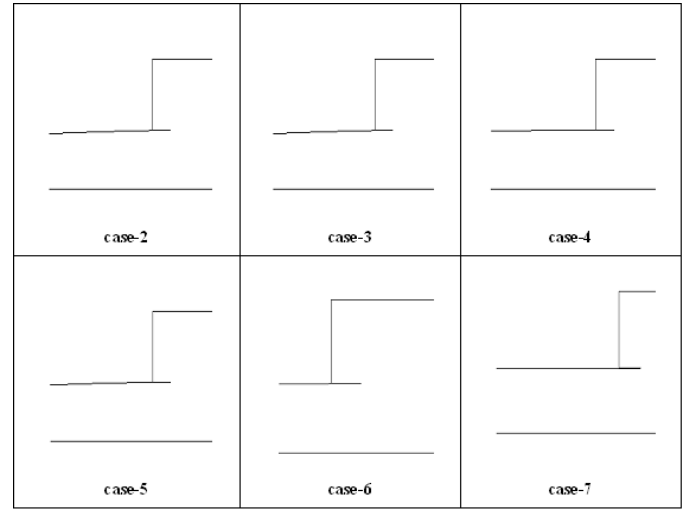

Figure 2. Nozzle curves of investigated cases

The final aim of these simulations is to find which one of these equivalent nozzles gives the closest results comparing with the real nozzle to be able to have a fairly good estimation of flow conditions at downstream parts of wind tunnel, meaning test section and diffuser, in conceptual design when the final nozzle curve is not ready to use.

\section{Governing Equations}

The governing equations (continuity, momentum, and energy) for a steady, compressible, non-isothermal and turbulent high-speed flow are as follows [7]:

$$
\begin{gathered}
\frac{\partial\left(\bar{\rho} \overline{\mathrm{u}}_{1}\right)}{\partial \mathrm{x}_{\mathrm{i}}}=0 \\
\rho\left(\frac{\partial \overline{\mathrm{u}}_{1}}{\partial \mathrm{t}}+\frac{\partial \overline{\mathrm{u}}_{\mathrm{i}} \overline{\mathrm{u}}_{\mathrm{j}}}{\partial \mathrm{x}_{\mathrm{j}}}\right)=-\frac{\partial \overline{\mathrm{p}}}{\partial \mathrm{x}_{\mathrm{i}}} \\
+\frac{\partial}{\partial \mathrm{x}_{\mathrm{j}}}\left[\mu\left(\frac{\partial \overline{\mathrm{u}}_{1}}{\partial \mathrm{x}_{\mathrm{j}}}+\frac{\partial \overline{\mathrm{u}}_{\mathrm{j}}}{\partial \mathrm{x}_{\mathrm{i}}}\right)-\rho \overline{\mathrm{u}_{1}^{\prime} \mathrm{u}^{\prime}}\right] \\
\nabla \cdot(\vec{v}(\rho E+p))=\nabla \cdot\left(k_{e f f} \nabla T+\tau_{e f f} \cdot \vec{v}\right)
\end{gathered}
$$

The equation of state (considering ideal-gas concept) is:

$$
\frac{\mathrm{p}}{\rho}=\mathrm{RT}
$$

\subsection{Turbulence Modeling}

According to the Boussinesq approximation [7], the Reynolds stresses can be related to the local velocity gradients by defining the turbulent eddy viscosity as in Eq. (5).

$$
\rho \overline{\mathrm{u}_{1}^{\prime} \mathrm{u}_{\mathrm{j}}^{\prime}}=\mu_{t} \frac{\partial \overline{\mathrm{u}_{1}}}{\partial x_{j}}
$$

A pair of turbulence scalar quantities, such as k- $\varepsilon$ ork- $\omega$ may be used to calculate the turbulent eddy viscosity. In the present study, the shear stress transport $\mathrm{k}-\omega(\mathrm{SST} / \mathrm{k}-\omega)$ model has been chosen.

The SST/k- $\omega$ turbulence model is a two-equation eddy-viscosity model. The use of ak- $\varepsilon$ formulation in the inner parts of the boundary layer makes the model directly usable close to the wall through the viscous sub-layer. 
Hence, the SST/k- $\omega$ may be used as a low-Reynolds turbulence model without any additional damping function. The SST formulation also switches to a $\mathrm{k}-\varepsilon$ behavior at fully turbulent flow fields and hence it avoids the common $\mathrm{k}-\omega$ problem where the model is too sensitive to the inlet free stream turbulence properties. The turbulence kinetic energy, $k$, and the specific dissipation rate, $\omega$, are represented by Eq. (6) and Eq. (7), respectively.

$$
\begin{aligned}
& \frac{\partial k}{\partial t}+\bar{u}_{\jmath} \frac{\partial k}{\partial x_{j}}=P_{k}-\beta^{*} k \omega \\
& +\frac{\partial}{\partial x_{i}}\left[\left(v+\sigma_{k} v_{t}\right) \frac{\partial k}{\partial x_{i}}\right] \\
& \frac{\partial \omega}{\partial t}+\bar{u}_{\jmath} \frac{\partial \omega}{\partial x_{j}}=P_{k}-\beta \omega+\frac{\partial}{\partial x_{i}}\left[\left(v+\sigma_{\omega} v_{t}\right) \frac{\partial \omega}{\partial x_{i}}\right]+ \\
& 2\left(1-F_{1}\right) \sigma_{\omega 2} \frac{1}{\omega} \frac{\partial k}{\partial x_{j}} \frac{\partial \omega}{\partial x_{j}}
\end{aligned}
$$

Thus, $\mu_{t}$ can be obtained from Eq. (8). Further details on the SST/k- $\omega$ model may be found in [7].

$$
\mu_{t}=\rho v_{t}=\frac{a_{1} k}{\max \left(a_{1} \omega, S F_{2}\right)}
$$

\section{Geometry and Boundary Conditions}

Fig. 1 shows the schematic view of computational domain. At the inlet region (left), the boundary condition is assumed as pressure inlet. No-slip and insulated conditions is considered for wall boundaries. The outlet boundary at the right is considered as pressure outlet. The flow and geometry is assumed to be axisymmetric due to save in computational costs.

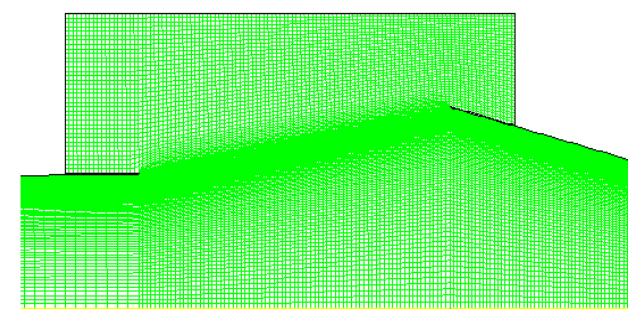

Figure 3. A typical view of grid distribution in the test section

A structured grid is used in computational domain for simulation that is dense near wall boundaries to detect details of solution correctly. A typical view of grid in the test section is shown in Fig. 3. The grid distribution has higher resolution near nozzle and diffuser walls to calculate boundary layer characteristics more accurately.

\section{UDF Calculations and Method of Analysis}

The inlet boundary conditions for all equivalent nozzles must be defined with the UDFs. The UDF of each case is defined in such a way that the exhausted condition would be similar to the real nozzle results. Two main parameters for comparing the cases are Mach number and boundary layer thickness. To reach the similar exhausted condition, it is necessary to define the inlet conditions truly. Hence the boundary layer thickness and Mach number are two main parameters for UDF that can be found with a try and error approach. Also, the ratio between boundary layer thickness of velocity and pressure must be considered. Static pressure is assumed to be constant at the inlet cross-section. The total pressure is set to be constant out of boundary layer region and varies exponentially in this region.

To study the flow field of ideal air in a steady turbulent flow, the continuity, momentum and ideal gas law are engaged and solved simultaneously. To find the turbulent flow statistics, the SST/k- $\omega$ formulation is used. It is a two-equation model that is based on eddy-viscosity concept. The density based solver is chosen for discretization of energy-momentum coupling. Also, the AUSM flux type is engaged with variable Courant number. The second-order discretization formulation is used for momentum, energy and turbulence equations. Since the static temperature varies in a wide range, the temperature dependence of dynamic viscosity is considered with Sutherland's law assumption.

\section{Results and Discussions}

Fig. 4 depicts the Mach number distribution at the test section entrance for all simulated cases. For case with real nozzle (case-1), the uniform Mach distribution can be seen at a little more than half of cross section that is must be considered in experimental tests. In the other word, more than $1 / 3$ of cross section is affected by boundary layer region. For all cases, a deflection point can be observed almost at the edge of boundary layer. Near the axis, the Mach number is equal for all cases, except case- 6 in which the Mach number is considerably less than others. Indeed, since case- 6 has a very low-length equivalent nozzle, there is not enough time for flow to modify itself and get a reasonable distribution at the end of equivalent nozzle. Case-3, case-4, and case-7 seem to have the minimum difference with case-1 among all cases.

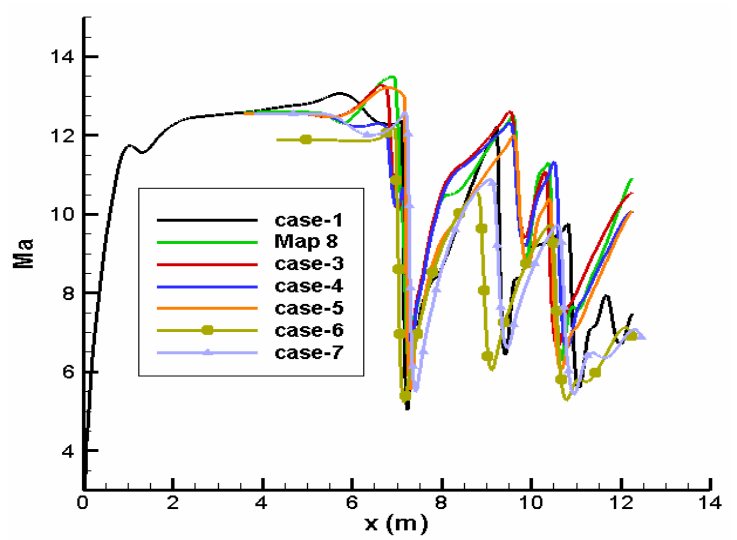

Figure 4. Distribution of Mach number along the test section entrance of wind tunnel 
Fig. 5 shows the radial variation of axial velocity for all different cases. One can see that the axial velocity profiles of all simulations are very close to that of real nozzle. In the contrary with the Mach distribution, the axial velocity distribution has no deflection point in all curves. Case-3 and Case- 4 have the best agreement with case- 1 comparing with other cases.

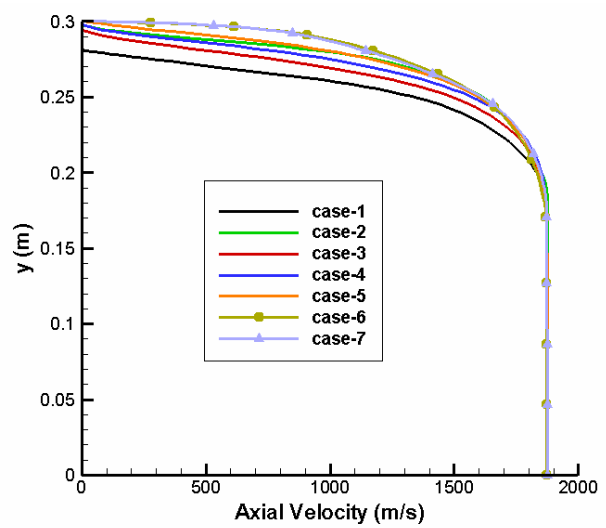

Figure 5.Distribution of velocity magnitude along the test section entrance of wind tunnel

The radial variation of total pressure at the entrance of test section is depicted if Fig. 6. The boundary layer thickness of total pressure is fairly considerable. It can be noted that the thickness of total pressure boundary layer is usually more than that of velocity and total pressure has a very low value near the wall. Getting far from the wall, its value is rising sharply. In addition, the total pressure curves for all cases have deflection points similar to Mach number distributions. Case-2, Case-6, and case-7 show the minimum difference with case-1.

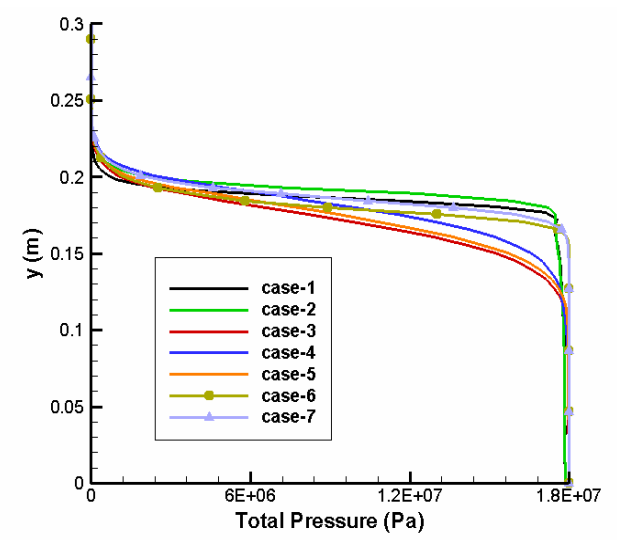

Figure 6. Distribution of total pressure along the test section entrance of wind tunnel

Fig. 7 depicts the radial variations of static pressure for all simulations at the nozzle exit. The value of static pressure at this cross-section is almost constant and is equal to a static pressure that is related with the Mach number and total pressure of the point on axis. The static pressure values of all equivalent nozzles are relatively equal to real nozzle, except case- 6 which shows about $40 \%$ difference with other cases. Indeed, this case has low-length that yields to low-expansion of flow and hence, the value of static pressure would be more that other cases.

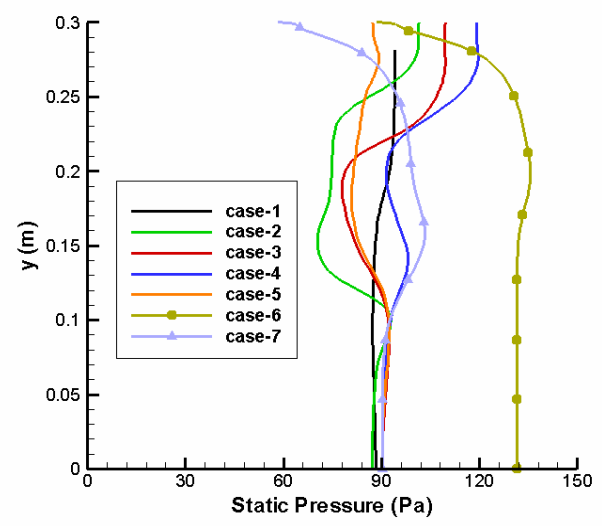

Figure 7.Distribution of static pressure along the test section entrance of wind tunnel

The axial derivative of Mach number $(\mathrm{dMa} / \mathrm{dx})$ is an important parameter for comparing the result of equivalent nozzles with the real one. Because this parameter conducts the flow with the specified acceleration into the test section while the velocities of all cases may be equal.

Fig. 8 demonstrates distribution of $\mathrm{dMa} / \mathrm{dx}$ along test section entrance. It is considerable that the $\mathrm{dMa} / \mathrm{dx}$ of real nozzle is fairly smaller than other equivalent nozzles. At the near wall region, the axial derivative of Mach number is positive for case-1 with real nozzle. This fact results in accelerating of the flow in the test section and therefore, the oblique shock of real nozzle would be greater and more powerful than other simulated cases. The values of $\mathrm{dMa} / \mathrm{dx}$ of cases 2, 3 and 4 are negative at the boundary layer that yields to deceleration of flow at downstream and consequently causes the weaker shocks, which will be discussed in Fig. 21. At the outer region of boundary layer, axial derivative of Mach number is almost zero for all cases meaning that the flow doesn't have any negative or positive accelerations. As seen in this figure, case-5 (and case-3) has the best agreement with case- 1 comparing with the other cases.

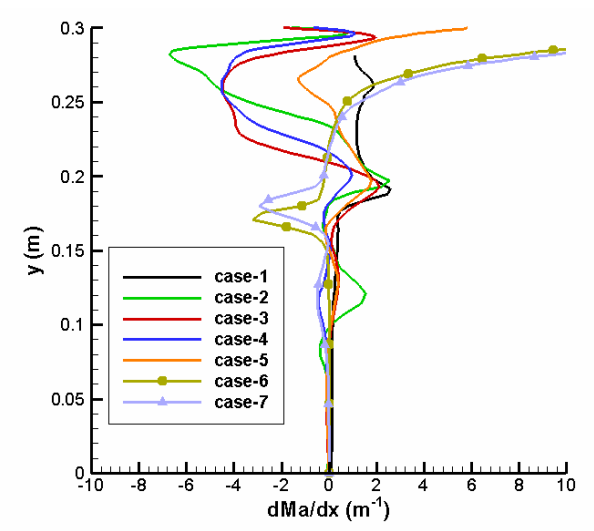

Figure 8.Distribution of Mach number axial derivative along the test section entrance of wind tunnel 
Fig. 9 shows the Contours of Mach number and static pressure for case-1. The flow ejected from nozzle is faced to a strong conic shock at the entrance of diffuser. Since the diffuser has a shock duct section, a shock system with some reflexes is formed in the diffuser. At the upper part of test section called "Dead-Zone", a low momentum flow can be seen created by recirculation of high momentum flow erupted from the nozzle. Due to viscous behavior of flow, a boundary layer region is created near all wall boundaries that can be observed as a low-Mach number region. Getting far from diffuser inlet, the Mach number of flow decreases by several relatively weak shocks called shock system in the diffuser throat part until it reaches the minimum level of itself at the end of diffuser. The shock system can be observed easily here that causes decrease in Mach number of flow while the static pressure is rising.

Fig.10 demonstrates the contours of total pressure and velocity magnitude in the computational domain for case- 1 . The level of total pressure remains constant in the nozzle and test-section parts (except at the boundary layer) because of isentropic characteristic of flow. When flow passes through the strong oblique shock at the inlet of diffuser, it loses its total pressure due to non-isentropic effects of the shock. It is noticeable that the oblique shock cannot be seen easily in this figure. Passing through a shock, the Mach number distribution has a great decrease while the value of velocity does not show any considerable change. This phenomenon can be explained by the fact that the Mach number is a variable of both velocity and temperature and hence, it is possible to Mach number to vary in a wide range while the velocity does not change considerably. At the divergent section of diffuser, a strong flow separation can be seen. The position of this separation depends on the diffuser outlet static pressure. By raising the value of outlet pressure, the separation region moves to upstream of the flow. The diffuser outlet static pressure for all simulated cases is adjusted such a way that the separation region remains at the entrance of divergent part of diffuser as seen in Fig.9.Contours of total pressure and velocity magnitude for case-1 are also shown in Fig.10. the strong shock in the entrance of diffuser can be indicated by a sharp decrease in total pressure. The shock system in the diffuser can be followed by following the total pressure contour. Two considerable recirculation zones can be seen in the contour of velocity magnitude; the first one is in the top of the test section and the second one is in the divergent part of the diffuser caused by a strong flow separation consequently occurs because of adverse pressure gradient in this region. These two recirculation zones can be depicted in the streamlines, shown in Fig.11.

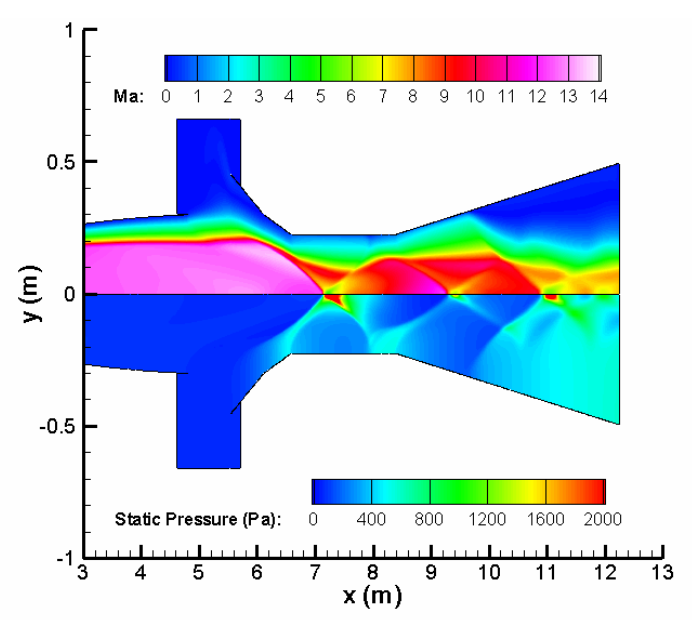

Figure 9.Contours of Mach number and static pressure for case-1

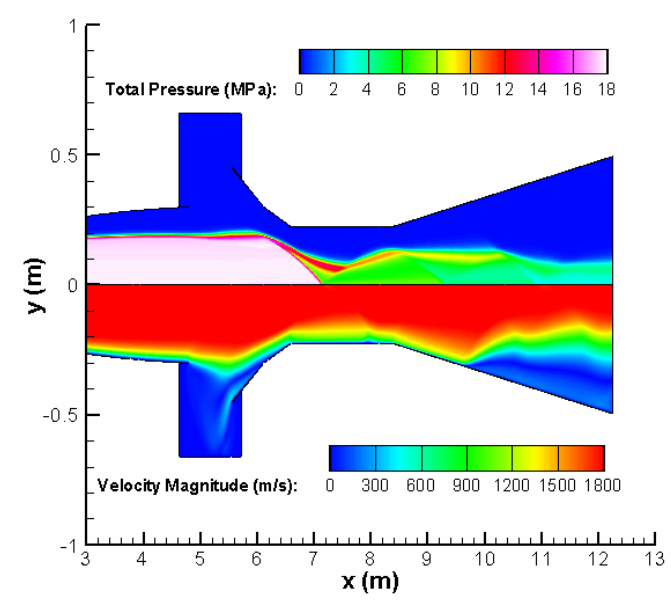

Figure 10. Contours of total pressure and velocity magnitude for case-1

Comparing Fig.9 and Fig. 10, it can be seen that the pair of Mach number and static pressure contours represents oblique shock characteristics better than pair of total pressure and velocity magnitude contours and hence, the first pair of contours is selected to study oblique shock system in diffuser for other simulated cases. In addition, a relatively strong flow separation can be observed at the one-third region of divergent part of the diffuser, caused by adverse pressure gradient.

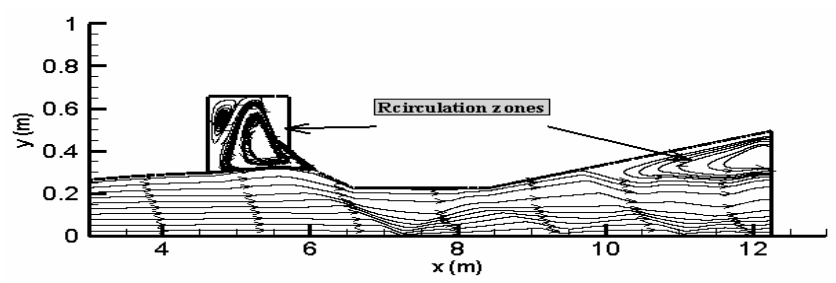

Figure 11.streamlines of case-1

Figures 12 to 17 depict the oblique shock system via contours of Mach number and static pressure for case- 2 to case-7, respectively. For all cases, the position of the first oblique shock on the axis is the same (near $\mathrm{x}=7 \mathrm{~m}$ ). Considering the difference between Mach number before 
and after shock, case-2 shows a little weaker shock comparing with case- 1 . Case- 3 is very similar to case- 2 . Results of case- 2 and case- 3 are very close to that of case- 1 at the test section and the entrance of diffuser while their difference with case- 1 tends to increase by getting far from the entrance of diffuser.

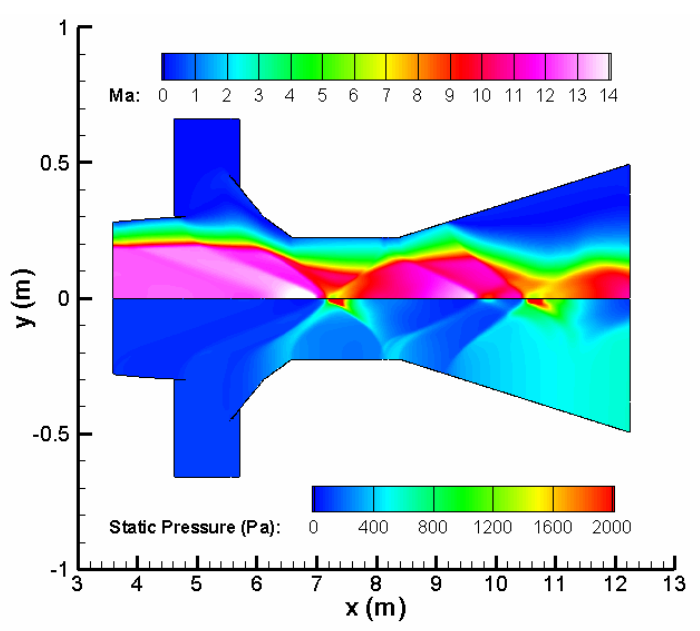

Figure 12. Contours of Mach number and static pressure for case-2

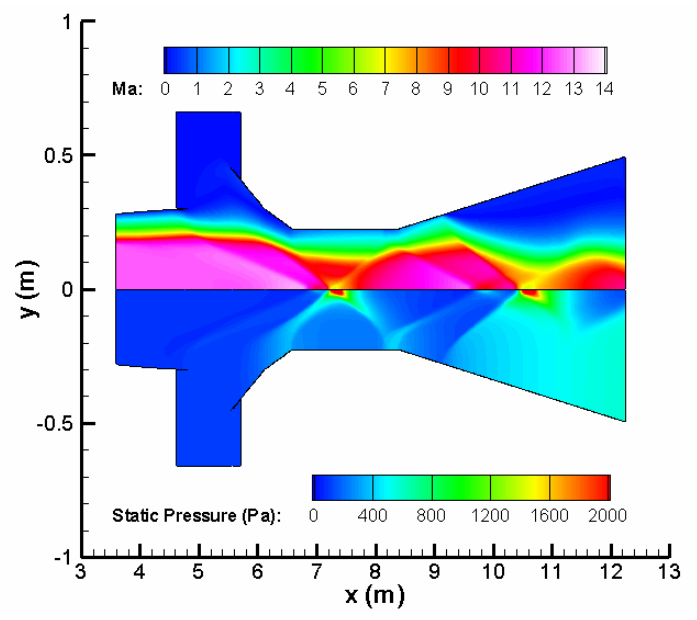

Figure 13. Contours of Mach number and static pressure for case-3

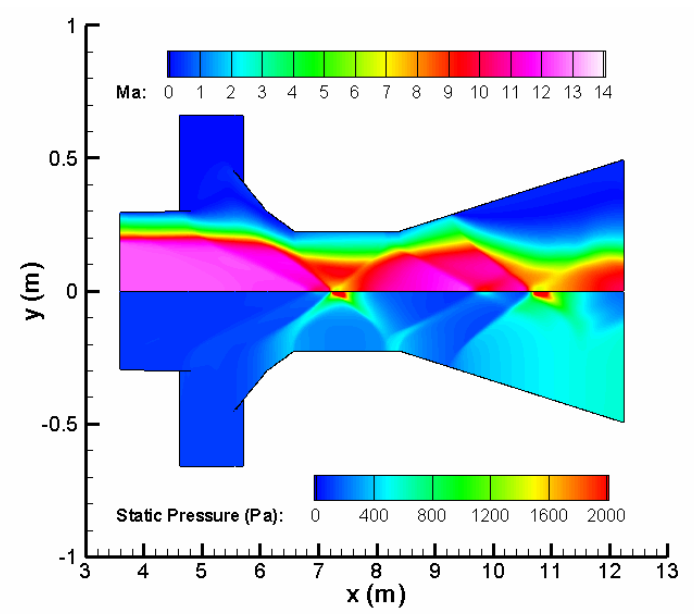

Figure 14. Contours of Mach number and static pressure for case-4

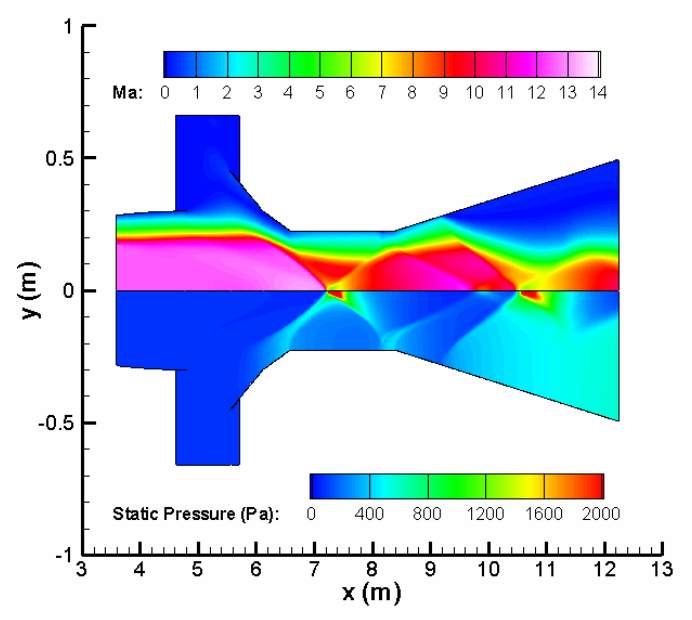

Figure 15. Contours of Mach number and static pressure for case-5

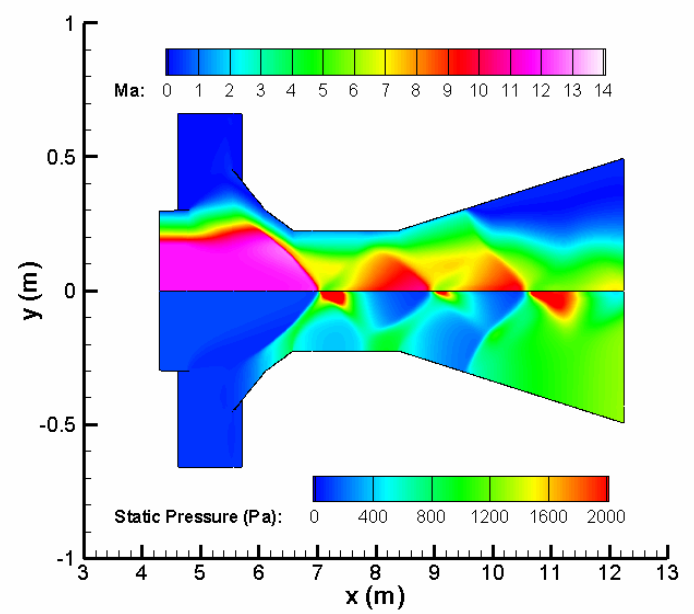

Figure 16. Contours of Mach number and static pressure for case-6

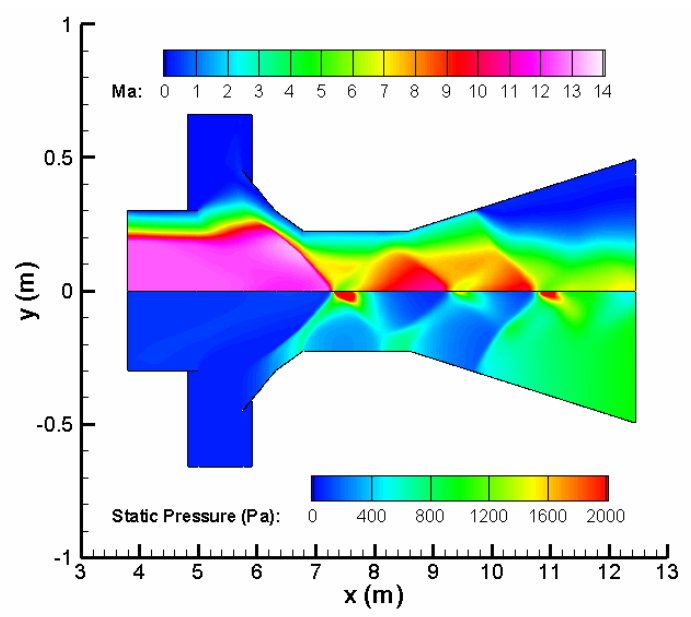

Figure 17. Contours of Mach number and static pressure for case-7

Results of case- 4 and case- 5 are also similar to case- 2 and case-3, but one can see a little variance between them at the end of diffuser. A high-Mach region can be observed around the axis for case- 1 to case- 5 . The oblique shock systems for case- 6 and case- 7 are totally different from other cases. In these two cases, the first oblique shock is 
very stronger than others and the high-Mach region around the axis no longer exists.

Fig. 18 depicts the variation of Mach number against axial location on the axis. Although this figure is very similar to Fig. 19 due to relation between Mach number and velocity, but it is considerable that by passing across the shock, a relative reduction of about $50 \%$ occurs in Mach number while the velocity magnitude decreases only about $5 \%$, as a consequence of enormous differences in temperature, static pressure and also density. It can be seen that the most similar Mach number distribution to real nozzle result (in the test section) is the result of case- 4 . According to Fig. 18, case-4 and case-3 have the most similarity with case- 1 in test section while case-7 and case- 6 are similar to case- 1 considering shock system.

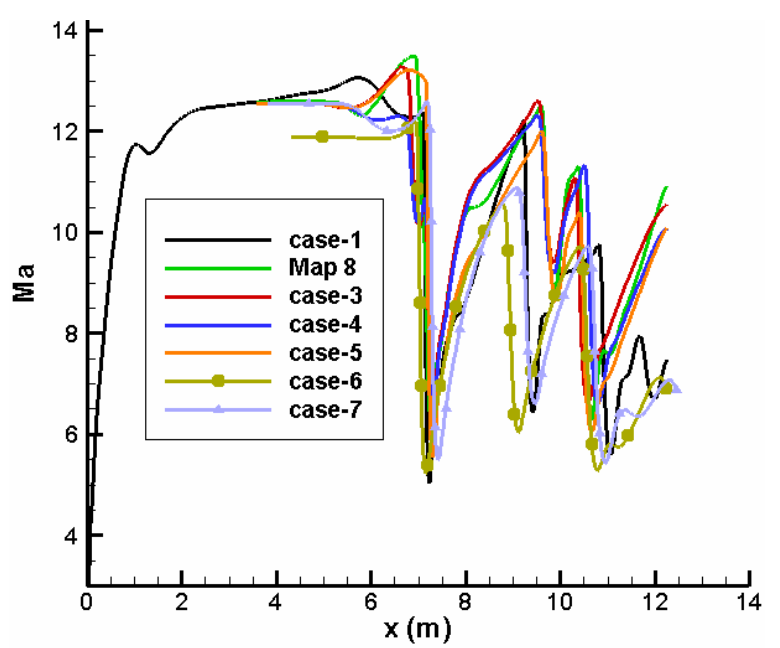

Figure 18.Distribution of Mach number along wind tunnel axis

Fig. 19 demonstrates the distribution of velocity magnitude versus axial coordinate at the axis. The most reduction in the velocity magnitude occurs in the region of shock creation. It is observed that the result of case-7and after that,case-6have the lowest difference with the real nozzle result.

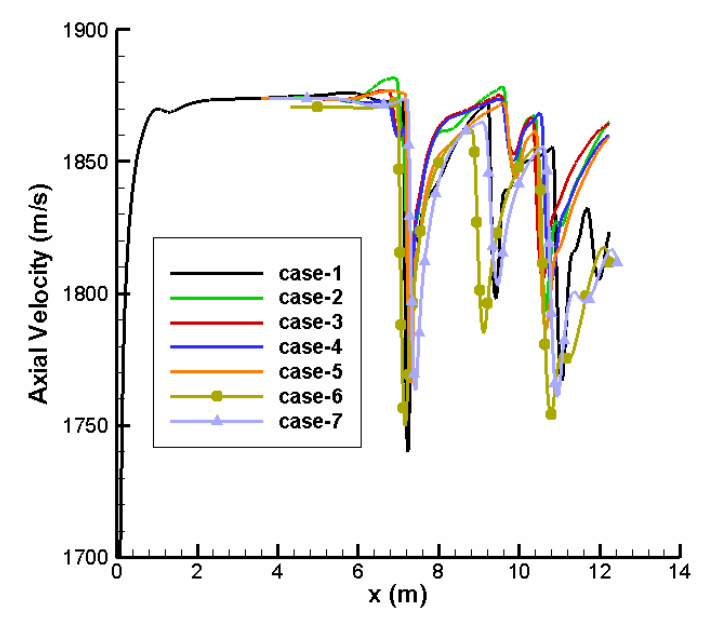

Figure 19.Distribution of velocity magnitude along wind tunnel axis
Fig. 20 shows the distribution of total pressure against axial distance from the inlet. The step-like behavior of curves can be explained by shock system that yields to reduction in the value of total pressure on the axis. A point that must be considered here is that all simulated cases predict decrease in total pressure of the first shock less that of real nozzle. Indeed, the result of real nozzle in this figure has an enormous difference comparing with all other cases. As the oblique shocks of cases 2,3 and 4 are weaker than case-1, the reduction in total pressure for this cases are smaller than of case-1, resulting in higher values of total pressure at the outlet for case 2,3 and 4, comparing with case-1.One can say that the closest result to real nozzle is case- 5 and after that, case- 6 and case- 7 can be mentioned.

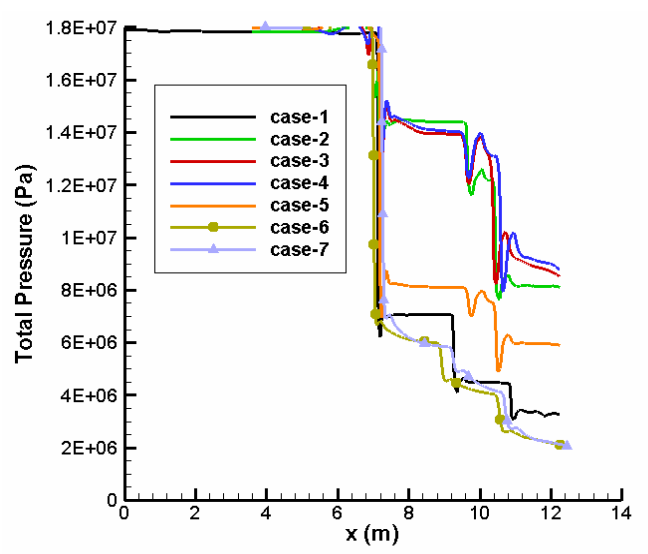

Figure 20.Distribution of total pressure along wind tunnel axis

Fig. 21 depicts the distributions of static pressure along axis for all seven simulated cases. All curves have a sharp increase in a point near $\mathrm{x}=7 \mathrm{~m}$ caused by the strong oblique shock in the diffuser entrance. Passing the diffuser throat, some other increases in all curves can be observed related to the shock system in this section. Getting far from the diffuser entrance, the differences between case- 1 and other cases results becomes more and more both in values of static pressure and the location where the maximum of static pressure occurs. One can say that the values of case-7 and after that,case-4and case- 6 have the closest result comparing with the result of real nozzle.

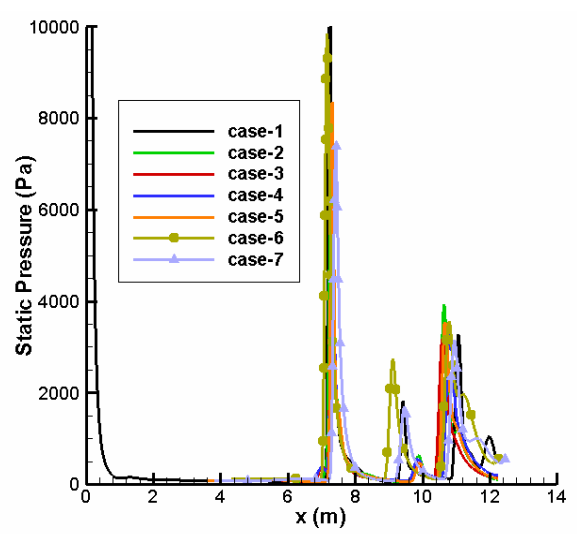

Figure 21.Distribution of static pressure along wind tunnel axis 
By considering all parameters distributions in the present manuscript, it can be concluded that the results of case- 4 can simulate the conditions of real nozzle better than other cases. Further detailed investigations must be conducted in this field to find the best entrance conditions for an undefined nozzle that can provide almost the same flow field for downstream parts of wind tunnel. Finally, by considering all of results, it can be concluded that case- 6 has the best similarity with the real nozzle among the simulated cases. It should be noted that this conclusion is only valid for this family of equivalent nozzles and a similar investigation should be carried out for other possible kinds of equivalent nozzles (e.g. conic nozzles).

\section{Conclusion}

A comparative study of equivalent nozzle has been carried out for the industrial high-speed wind tunnel. The equivalent nozzle design is needed in the conceptual and primary design process of wind tunnel, especially when the curve nozzle is undefined and must be calculated during designing process. The equivalent nozzle can provide a relatively good flow condition downstream of the nozzle-exit (for test section, diffuser and etc.) and can help thedesigner for primary designing these parts of wind tunnel without needing real nozzle curve. Also, if the pattern of flow is similar in the test section for real and equivalent nozzles, the study of the tested model can be carried out easily in the primary estimations.

In the present study, several cases of equivalent nozzles have been simulated and examined to find that which one has the best results comparing with the real nozzle data. The comparison should be divided into two regions, test section and diffuser. In the test section region, case- 3 and after that, case- 4 show the closest result to the results of real nozzle and they can be useful for flow investigation around the tested model in the test section. On the other hand, the $1.2 \mathrm{~m}$-length Ogive curve with 0.5 degree initial angle and after that, the case of 1.2 m-length duct, case-7 have the best agreement with real nozzle in simulating of flow and calculating of shock system and they can be used for diffuser designing. It should be noted that this conclusion is only valid for this family of equivalent nozzles and a similar investigation should be carried out for other possible kinds of equivalent nozzles.

\section{Nomenclature}
$\mathrm{d} / \mathrm{dx} \quad$ Axial Derivative
E Internal Energy (J)
k Turbulence kinetic energy $\left(\mathrm{m}^{2} / \mathrm{s}^{2}\right)$

$\begin{array}{ll}\mathrm{k}_{\mathrm{eff}} & \text { Effective thermal conductivity }(\mathrm{W} / \mathrm{m}-\mathrm{K}) \\ \mathrm{Ma} & \text { Mach Number } \\ \mathrm{p} & \text { Static Pressure }(\mathrm{Pa}) \\ \mathrm{T} & \text { Temperature }(\mathrm{K}) \\ \mathrm{u} & \text { Velocity }(\mathrm{m} / \mathrm{s}) \\ \bar{u}_{\imath} & \text { Mean velocity components for turbulent velocity }(\mathrm{m} / \mathrm{s}) \\ u^{\prime} & \text { Fluctuating components for turbulent velocity }(\mathrm{m} / \mathrm{s}) \\ \vec{v} & \text { Velocity vector }(\mathrm{m} / \mathrm{s}) \\ \mathrm{x} & \text { Radial coordinate }(\mathrm{m}) \\ \mathrm{y} & \text { Axial coordinate }(\mathrm{m}) \\ \alpha & \text { Thermal diffusivity }\left(\mathrm{m}^{2} / \mathrm{s}\right) \\ \varepsilon & \text { Dissipation rate of turbulence kinetic energy }\left(\mathrm{m}^{2} / \mathrm{s}^{3}\right) \\ \mu & \text { Dynamic viscosity }(\mathrm{Kg} / \mathrm{m}-\mathrm{s}) \\ \mu_{t} & \text { Turbulent eddy viscosity }(\mathrm{Kg} / \mathrm{m}-\mathrm{s}) \\ \rho & \text { Density }\left(\mathrm{Kg} / \mathrm{m}^{3}\right) \\ \tau_{e f f} & \text { Effective shear stress }(\mathrm{Pa}) \\ \omega & \text { Turbulence eddy frequency }\left(\mathrm{s}^{-1}\right) \\ & \end{array}$

\section{References}

[1] J.M.A.Longo,"Modeling of high-speed flow phenomena," RTO AVI Lecture series on Critical Technologies for High-speed Vehicle Development, Belgium, May, 2004.

[2] R.E. Deem, "Pressure and heat-transfer distribution tests on a standard ballistic-type model HB-2 at mach 2 to 5 , including the effects of shock impingement," Report S-R6, Douglas Aircraft Company, California, USA, Jun, 1963.

[3] J.D. Gray, and E. E. Lindsay, "Summary report on aerodynamic characteristics of standard model HB-1 and HB-2," Technical report AEDC-TDR-63-137, Arnold engineering development center, USA, 1964.

[4] S. Kuchi-Ishi, And E. Watanabe, "Comparative force/heat flux measurements between JAXA hypersonic test facilities using standard model HB-2 Part 1\&2," JAXA research and development report, Japan Aerospace Exploration Agency, Japan, 2005.

[5] J.D.Anderson, "Modern compressible flow with historical perspective," McGraw-Hill Inc., New York, 2003.

[6] Group of writers, "Handbook of supersonic aerodynamics," Vol.6. Sec.17 Ducts, Nozzles and Diffusers, NAVWEPS Report 1488, 1964.

[7] B. E. Launder, N. D. Sandham, "Closure strategies for turbulent and transitional flows," Cambridge University Press, Cambridge, UK, 2002. 